\title{
Systematic bias in real-world tonometry readings based on laterality?
}

\author{
John C. Buchan $\mathbb{1 0}^{1,2} \cdot$ David Macleod $^{2} \cdot$ William Hickman ${ }^{3} \cdot$ Andrew Bastawrous $^{2}$
}

Received: 13 March 2019 / Revised: 27 June 2019 / Accepted: 5 July 2019 / Published online: 9 August 2019

(c) The Author(s), under exclusive licence to The Royal College of Ophthalmologists 2019

\begin{abstract}
Aims In research settings, the first eye examined tends to have a higher intraocular pressure (IOP) than the second. We sought to verify whether clinicians in Yorkshire, UK, measure IOP in right eyes before left and whether such behavioural factors affect IOP readings at the population level.

Methods We observed 128 IOP measurements taken by 28 ophthalmologists using Goldmann applanation tonometry (GAT) over a 4-month period in 2018, recording which eye was examined first. All IOP measurements on electronic patient records for Leeds Teaching Hospitals NHS Trust, UK, between January 2002 and June 2017 were extracted, yielding IOP readings for 562,360 eyes, analysed for evidence of systematic bias in IOP measurement.

Results Right eye IOP was measured before left in 112/128 observations (87.5\% (95\% CI: 75.2\%-94.2\%)). For IOP measured by GAT, there was no statistically significant difference $(p=0.121)$ between right and left eye IOP (mean IOP 16.95 and $16.96 \mathrm{mmHg}$, respectively). Even values of IOP were reported more frequently than odd values (136,503/214,628 $(63.6 \%)$ were even). Identical IOP readings for both eyes were recorded in 124,392/254,380 patients (48.9\%) who had both eyes measured.

Conclusions Our study found no IOP difference based on laterality, but strong evidence of certain trends associated with IOP measurement by GAT, such as a preference for even values and the same IOP being recorded for both left and right eyes. Such effects may be explained by behavioural aspects of GAT and suggest that there are substantial opportunities for improvement in the way GAT is utilised in real world settings.
\end{abstract}

\section{Introduction}

Glaucoma is the third most common cause of blindness globally after cataract and uncorrected refractive error, and despite increases in understanding of the aetiology, intraocular pressure (IOP) remains the primary modifiable risk factor for progressive glaucomatous visual loss [1]. In undertaking a large population-based cohort study in Nakuru, Kenya, which included measurement of participants' IOP, it was noted that the IOP of right eyes were significantly higher than the fellow left eyes [2-4]. This same observation has been described by other studies that

\footnotetext{
John C. Buchan

John.Buchan@1shtm.ac.uk

Leeds Teaching Hospitals NHS Trust, Leeds, UK

2 London School of Hygiene and Tropical Medicine, London, UK

3 Hull University Teaching Hospitals NHS Trust, Hull, UK
}

report IOP difference between right and left eyes [5-8], despite no known physiological difference between right and left eyes that could explain this difference. If this research finding were also present in routine clinical practice, then a systematic bias in IOP measurement could lead to a systematic overtreatment of right eyes relative to left, which at a population level may have implications for clinical outcomes and resource allocation.

The possibility of publication or reporting bias exists, in that it would be of little interest to report the finding that no difference was found in the IOP readings of right and left eyes in a population. However, a prospective study prompted by the statistically significant finding from the Ocular Hypertension Treatment Study (OHTS) that right eyes were 0.3 (SD $+/-2.8) \mathrm{mmHg}$ more hypertensive than left demonstrated that IOP is measured higher in the first eye examined, regardless of whether that is the left or right eye [9].

The reason for the first examined eye being measured as having a higher IOP than the second eye is conjectured to relate to patients squeezing their lids or inadvertently 
performing a Valsalva manoeuvre as they hold their breath for the first eye, which have both been shown to elevate IOP $[9,10]$. Patients squeezing their eyes during tonometry has been shown to reduce with subsequent IOP readings [9], which offers an explanation for the relatively lower second eye IOP measurement. The reduced squeezing/Valsalva at second eye measurement would be compounded by the fact that the elevated IOP during squeezing is expected to increase ocular outflow, thereby tending the second examined eye to have a lower IOP on relative relaxation.

As with OHTS, the directionality of the difference in IOP (right $>$ left) from the Nakuru data could be explained by the examination protocol that stipulated that right eyes were to be examined first [2]. The existence of corroborating studies reporting this same finding, and the absence of conflicting results with no studies found identifying left IOPs higher than right, is postulated to be an artefact of the prevailing culture within clinical ophthalmology and ophthalmic research to examine right eyes first. There is no published evidence, to our knowledge, of the level of adherence of ophthalmologists to the perceived cultural norm of examining right eyes before left eyes, and no published report demonstrating the extent to which IOP readings between right and left eyes differ in routine clinical practice.

We determined to verify, by opportunistic observation of clinicians performing tonometry, whether ophthalmologists in Yorkshire (UK) routinely examine right eyes prior to left in their day-to-day practice. We also sought to evaluate whether this cultural practice, if verified, has implications for right and left IOP readings over a large population.

\section{Subjects and methods}

To test the perception that ophthalmologists are habituated to check the IOP in the right eye first, three ophthalmologists from Leeds Teaching Hospitals NHS Trust (LTHT) recorded, in the passage of their daily work, which eye was examined first by any colleague observed using any form of tonometry over a 4-month period in 2018. They recorded the grade of the clinician being observed to differentiate those within the 7-year ophthalmic training programme termed "trainees" and those termed "senior", being in career positions (consultant, staff grade or associate specialist). The method of tonometry was recorded being divided between Goldmann applanation tonometry (GAT), rebound tonometry with iCare (Icare Oy, Vanda, Finland), and air puff using Reichert tonometer (Reichert Technologies, Buffalo, NY, USA). The GAT is a manual, analogue device whilst the rebound and air puff tonometers are semi-automated, digital devices. It was recorded which eye was measured first, and whether the clinician then went back to the first eye again to recheck IOP a second time.
Search was performed of the electronic patient record (Medisoft Ltd, Leeds, UK) for LTHT between January 1, 2004 and August 31, 2016 including any IOP reading with applanation, air puff or rebound tonometry. Statistical analysis was performed, and all figures generated using Stata (StataCorp. 2017. Stata Statistical Software: Release 15. College Station, TX: StataCorp LLC). IOP for each eye was recorded and the electronic patient record enforces recording of method of testing. This is a large teaching hospital, so includes specialist clinics such as glaucoma clinics, but local referral patterns mean that there is not a large burden of tertiary referral patients with complex glaucoma. The case mix at this hospital, therefore, closely reflects the ophthalmic needs of the catchment population, and can be taken as a representative of a typical case mix of an UK hospital providing comprehensive ophthalmic services.

Ethical approval for the Nakuru cohort study was obtained as reported previously [2], and separate ethical approval from the London School of Hygiene and Tropical Medicine Research Ethics Committee was obtained for the observations from Yorkshire.

For the observations of ophthalmologists, the proportion of examinations where the right IOP was the first to be measured (without subsequent return to this eye for repeat testing) was estimated. The confidence interval was adjusted to allow for the clustering of observations by ophthalmologist, as some individuals were observed multiple times.

From the electronic patient record, IOP summary statistics were reported along with the distributions of IOP illustrated by IOP measurement method. Two other sources of bias beyond the differences between right and left eyes were described; the proportion of even and odd number values and the proportion of pairs of eyes with exactly the same IOP in each eye. A paired $t$-test was performed to identify any systematic bias in the IOP between eyes. The expected proportion of even and odd values for IOP was $50 \%$, so a one-sample $Z$-test was used to test whether the proportion was different from this.

\section{Results}

\section{Practice of ophthalmologists in Yorkshire}

Twenty-eight ophthalmologists (12 senior and 16 trainees) were observed during 128 patient eye examinations ( 44 by senior and 84 by trainee ophthalmologists), checking the IOP using GAT on both eyes between January 3, 2018 and April 30, 2018.

Of the 128 observations, 112 recorded the right eye being checked first without any return to the first eye for 
(a) GAT

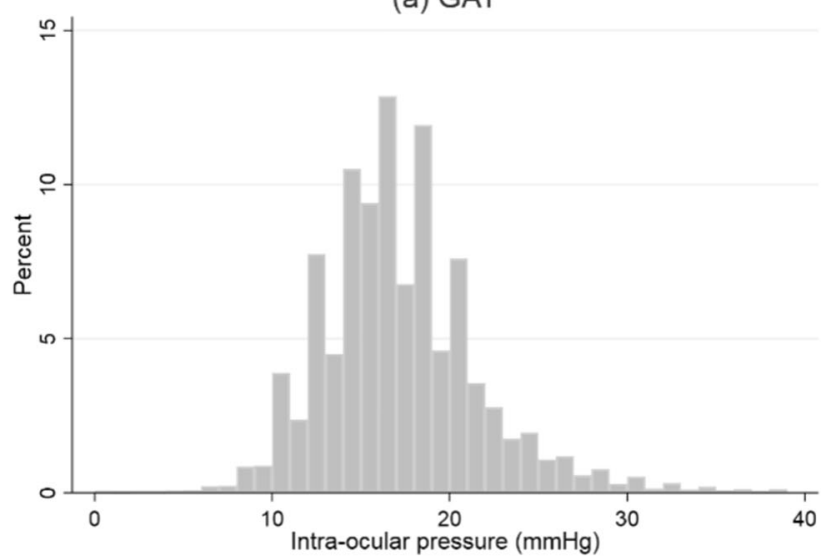

(c) Rebound

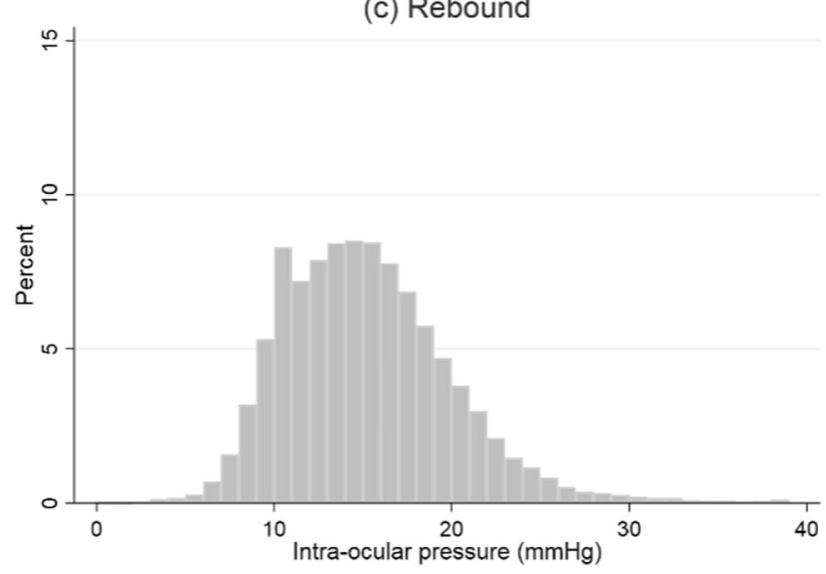

(b) Air-puff

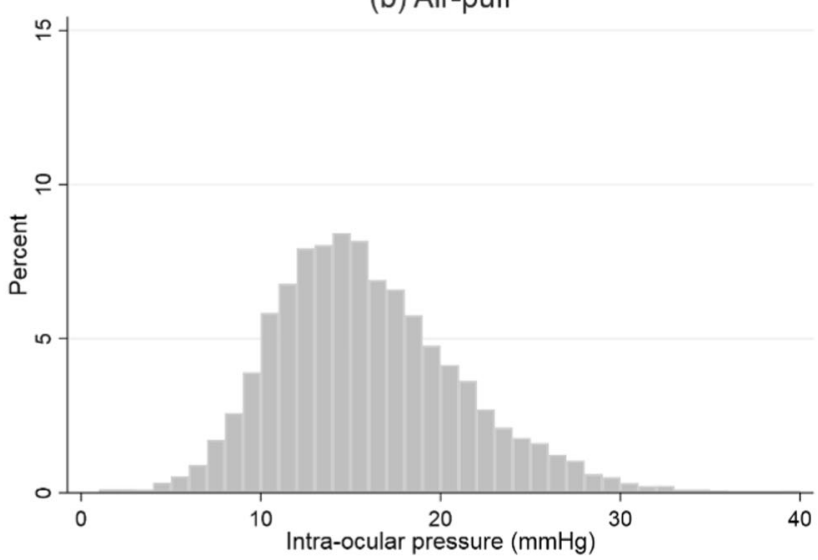

Fig. 1 Histogram of IOP for each measurement method a GAT, $\mathbf{b}$ air puff and $\mathbf{c}$ rebound. The graphs are curtailed at $40 \mathrm{mmHg}$ as each have a few extreme observations

repeat testing, resulting in an estimated prevalence of this practice of $87.5 \%$ (95\% CI: $75.2 \%-94.2 \%$ ).

Among observations of senior ophthalmologists, 95.5\% of examinations followed this practice, compared with $83.3 \%$ of those by trainees. This suggests a trend toward increased adherence to the perceived cultural norm with seniority, however, evidence of a true difference between these groups is weak $(p=0.125)$.

\section{Leeds IOP data results}

The IOP of 562,360 eyes were obtained from 308,044 patients aged 0-114 years, attending LTHT between January 1, 2002 and June 30, 2017. The majority of patients $(85.7 \%)$ had their IOP measured using GAT, with the remaining patients having their IOP measured using the rebound method $(11.7 \%)$ or air puff $(2.6 \%)$.

Overall, mean IOP in the eyes measured was $16.6 \mathrm{mmHg}$ (SD $5.0 \mathrm{mmHg}$ ). Where GAT was used, the mean IOP was $16.9 \mathrm{mmHg}$ (SD 4.9), in rebound $14.9 \mathrm{mmHg}$ (SD 5.4) and air puff $15.9 \mathrm{mmHg}$ (SD 5.5).
Comparing right and left IOP where GAT was used (and restricting to only the 214,628 who had IOP in both eyes recorded using GAT) found a mean IOP for right eyes of $16.95 \mathrm{mmHg}$ (SD 4.9) and left eyes mean IOP of 16.96 $\mathrm{mmHg}$ (SD 4.8). Resulting in an observed difference of $0.01 \mathrm{mmHg}$ (left IOP higher than right) but no evidence of a true systematic difference between eyes $(p=0.121)$.

The distributions of IOP were right skewed (Fig. 1a-c) and when using GAT, a clear even digit preference was observed (Fig. 1a). For GAT measurements, even values of IOP were reported with greater frequency than odd (136,503/214,628 (63.6\%) of observations were even, $p<$ 0.0001 from one-sample Z-test). No material difference between odd and even numbers was observed when either of the two electronic measuring techniques were used (49.9\% even values with air puff, $50.7 \%$ even with rebound).

There were 254,380 patients who had the IOP of both right and left eyes measured. The distribution of the difference in the IOP in the right and left eyes (IOP in right eye minus IOP in left eye) for each measurement is shown in 
(a) GAT

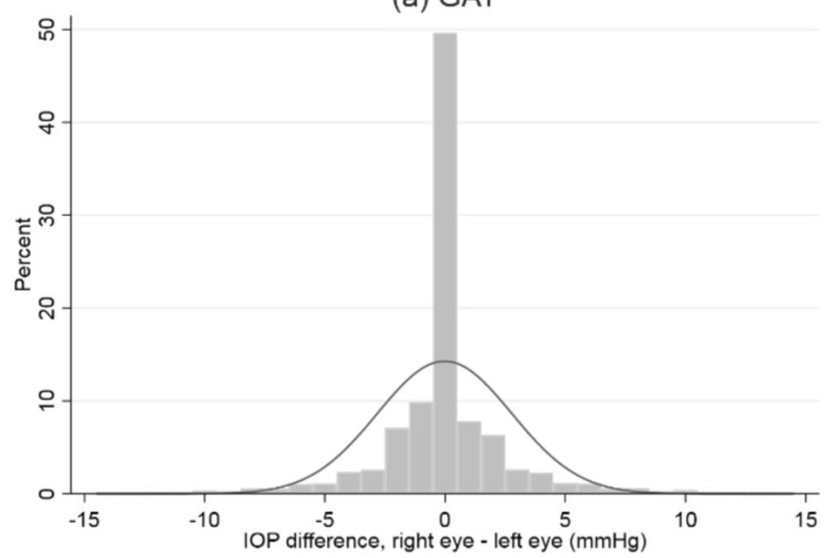

(c) Rebound

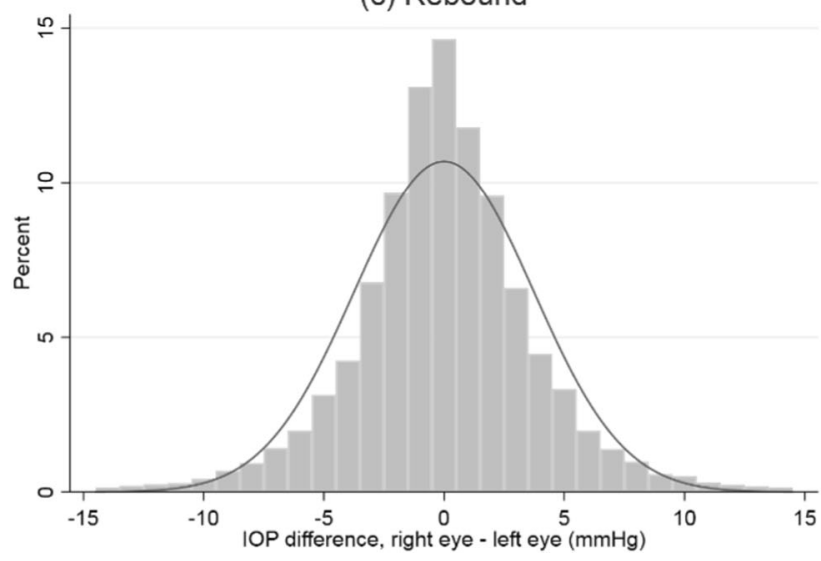

Fig. 2 Histogram of differences in IOP between individuals' eyes (right eye-left eye) IOP for each measurement method a GAT, b air puff and $\mathbf{c}$ rebound. A curve representing the expected distribution if

Fig. 2a-c. When using air puff or rebound tonometry (Fig. 2b, c) the data were normally distributed. However, when using GAT there were far more patients with an identical IOP recorded in both eyes than would be expected (Fig. 2a), in fact 124,392/254,380 patients (48.9\%) of patients had the same IOP in each eye.

\section{Discussion}

With repeated published studies showing that, under research protocol conditions, the first eyes measured have systematically higher pressures than the second eyes measured, it was considered an important research question to see if this same effect was at play in routine clinical practice. Our assumption that there is a prevailing cultural norm within ophthalmology to examine right eyes first was borne out by observations of colleagues measuring IOP, with seven out of every eight measurements following that pattern, and although evidence of a difference between senior

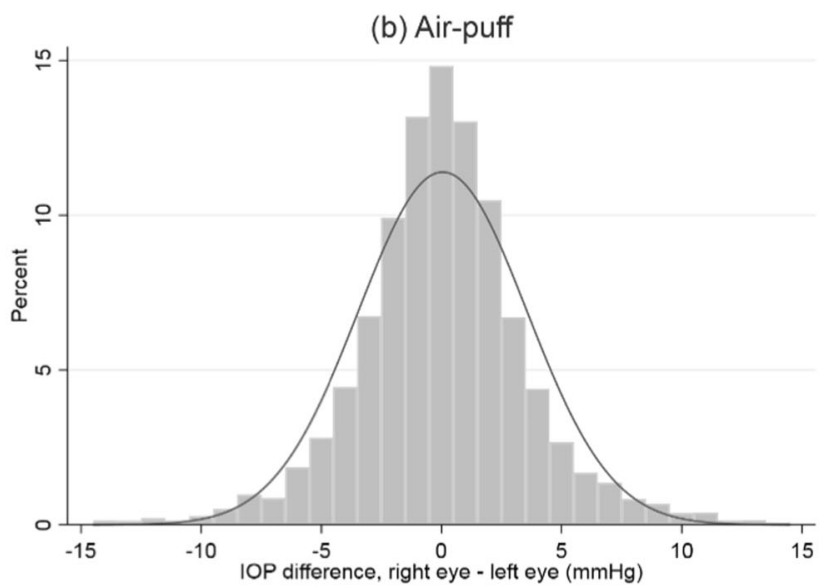

the data were normally distributed around zero is overlaid. Graphs are curtailed at $+/-15 \mathrm{mmHg}$

and more junior ophthalmologists was weak, the seniors were observed more often to adhere to this unwritten rule.

The effect of this cultural norm on the population of right and left eye IOP readings was evaluated by examining routinely collected data from over half a million readings on our electronic patient record-and no meaningful difference between right and left measurements was found. GAT is the established gold-standard method of IOP measurement, with some well described sources of error[11], however, analysis of our data has raised more questions than anticipated.

A very strong preference for even numbers was found with GAT, being recorded with almost double the frequency of odd numbers (63.6\% versus $36.4 \%)$. Non-human IOP measuring methods (rebound and air puff) did not exhibit this same even number preference, clearly indicating that there is a substantial behavioural element to our measurement of IOP with GAT or recording thereof. This even number preference has been demonstrated before to a very modest extent in a 1966 population-based study [12]. 
Further demonstration of behavioural biases is given by the fact that around half of all pairs of readings using GAT had both right and left eyes with exactly the same IOP. It should be expected that plotting a graph of the difference between right and left eye IOPs would form a normal distribution centred around zero (which is what was observed in the non-human measuring methods); or, if we factor in the effect of first eye measurement being higher than second as demonstrated in RCT and previous epidemiological surveys, we could expect a normal distribution centred around $0.3 \mathrm{mmHg}$. However, the distribution is far from normal, which again suggests that factors other than "the actual IOP" influence the recorded IOP. Candidate explanations for the "even number preference" and the "sameIOP preference" found with GAT centre around the way that IOP is tested with GAT in real-life settings.

In a research setting, IOP testing protocols are set out that dictate a highly standardised method, such as "with one examiner measuring the IOP and rotating the tonometer dial and a second examiner reading and recording the IOP measurement from the tonometer dial. The tonometer dial was rotated to $10 \mathrm{mmHg}$ prior to all measurements." [9] A description of real-world IOP measurement might describe "testing of the right eye first with the dial starting at whatever IOP reading the previous patient happened to have had; a mental note is then taken of the nearest even number to the dial reading before moving to the left eyewhich if it is approximately the same will be recorded as such, but if the mires are far apart then the dial will be adjusted to the best-fit even integer".

The observations in this study might encourage further exploration of the extent to which ophthalmologists can be encouraged to adopt research quality protocols in their daily IOP checking routines. The argument for the continued promotion of GAT in clinical practice is that glaucoma research has, for decades, been based on GAT - and all guidelines and treatment thresholds are based on this GAT driven data. The inference is that clinical decisions based on that research must therefore also utilise GAT. However, our study suggests that what is occurring in research settings and what is occurring in real-life clinics, whilst both being referred to as GAT, are not the same thing. "Real-world GAT" may be no more similar to "Research-GAT" than the rebound and air puff techniques, which were not found to have the same problems with biases and might therefore not be as inferior in routine practice as is sometimes suggested. Better adherence to gold-standard behaviour in GAT would be expected to be totally achievable but requires inculcation from the inception of ophthalmic training to create good habits that can last a career.

Promotion of the use of GAT that more closely adheres to research standards would be the preferred option, as although a move towards increased utilisation of non- human methods of IOP measurement would eliminate behavioural biases, it would require extensive investigation of the validity of applying management principles established by GAT-based research to clinical practice based upon another method of IOP measurement.

\section{Summary}

\section{What was known before}

- In research settings, the first eye measured will tend to have a higher IOP than the second eye. There is the perceived medical norm to examine the right eye of patients prior to the left eye.

\section{What this study adds}

- Ophthalmologists generally adhere to the unwritten rule that we examine right eyes first. Despite this, in a large sample of real-world IOP readings there was no difference between the right and left eye pressure measurements. Substantial behavioural biases were demonstrated in IOP reading using GAT with a strong preference to record even numbers, and to record identical IOP in right and left eyes.

Acknowledgements JCB is supported by a grant from the Queen Elizabeth Diamond Jubilee Trust to the Commonwealth Eye Health Consortium at the International Centre for Eye Health, London School of Hygiene and Tropical Medicine.

\section{Compliance with ethical standards}

Conflict of interest The authors declare that they have no conflict of interest.

Publisher's note: Springer Nature remains neutral with regard to jurisdictional claims in published maps and institutional affiliations.

\section{References}

1. Flaxman SR, Bourne RRA, Resnikoff S, Ackland P, Braithwaite $\mathrm{T}$, Cicinelli MV, et al. Global causes of blindness and distance vision impairment 1990-2020: a systematic review and metaanalysis. Lancet Glob Health. 2017;5:e1221-34.

2. Bastawrous A, Mathenge W, Peto T, Weiss HA, Rono H, Foster A, et al. The Nakuru eye disease cohort study: methodology \& rationale. BMC Ophthalmol. 2014;14:60.

3. Bastawrous A, Mathenge W, Wing K, Rono H, Gichangi M, Weiss HA, et al. Six-year incidence of blindness and visual impairment in Kenya: the Nakuru eye disease cohort study. Invest Ophthalmol Vis Sci. 2016;57:5974-83. 
4. Bastawrous A, Mathenge W, Buchan J, Kyari F, Peto T, Rono H, et al. Glaucoma features in an East African population: a 6-year cohort study of older adults in Nakuru, Kenya. J Glaucoma. 2018;27:455-63.

5. Bhorade AM, Gordon MO, Wilson B, Weinreb RN, Kass MA, Ocular Hypertension Treatment Study G. Variability of intraocular pressure measurements in observation participants in the ocular hypertension treatment study. Ophthalmology. 2009;116:717-24.

6. Liu JH, Sit AJ, Weinreb RN. Variation of 24-hour intraocular pressure in healthy individuals: right eye versus left eye. Ophthalmology. 2005;112:1670-5.

7. Vernon SA, Jones SJ. Intraocular pressure asymmetry in a population tested with the Pulsair non-contact tonometer. Eye. 1991;5:674-7.
8. Bankes JL, Perkins ES, Tsolakis S, Wright JE. Bedford glaucoma survey. Br Med J. 1968;1:791-6.

9. Pekmezci M, Chang ST, Wilson BS, Gordon MO, Bhorade AM. Effect of measurement order between right and left eyes on intraocular pressure measurement. Arch Ophthalmol. 2011;129:276-81.

10. dos Santos MG, Makk S, Berghold A, Eckhardt M, Haas A. Intraocular pressure difference in Goldmann applanation tonometry versus Perkins hand-held applanation tonometry in overweight patients. Ophthalmology. 1998;105:2260-3.

11. Whitacre MM, Stein R. Sources of error with use of Goldmanntype tonometers. Surv Ophthalmol. 1993;38:1-30.

12. Hollows FC, Graham PA. Intra-ocular pressure, glaucoma, and glaucoma suspects in a defined population. $\mathrm{Br} \mathrm{J}$ Ophthalmol. 1966;50:570-86. 